\title{
PISA Fen, Matematik ve Okuma Puanları Arasındaki Bağımlılık Yapısının Kapula ile Modellenmesi
}

\author{
Mervenur PALA*, Fatih SAĞLAM
}

\author{
Ondokuz Mayıs Üniversitesi, Fen Edebiyat Fakültesi, İstatistik Bölümü, SAMSUN
}

\author{
* mervenur.pala1@gmail.com
}

\begin{abstract}
Öz: PISA, bir ülkedeki fen, matematik ve okuma alanlarında öğrencilerin eğitim seviyelerini ölçen güvenilir bir araştırmadır. Bu bağlamda ülkelere ait farklı alanlarda (fen-matematik-okuma) puanlar elde edilerek, ülkelerarası eğitim düzeylerini karşılaştırmak ve ileriye yönelik olarak eğitim politikalarının belirlenmesi amaçlanmıştır. PISA'nın ulusal düzeyde çeviri ve uyarlama işlemleri, analizlerinin yapılması ve ulusal raporun hazırlanması, araştırmaya katılan her ülke için belirlenen ulusal merkezler tarafindan yürütülmektedir. Kapulalar, değişkenler arasındaki bağımlılık yapısını ortaya koyan fonksiyonlar olup, bununla beraber iki ya da çok değişkenli dağılımları oluşturur. Kapula fonksiyonunun asıl amacı, gözlenen verilere en uygun düşen çok değişkenli dağılımı, bağımlılık yapısını da ortaya koyarak elde etmektir. PISA, bireylerin eğitim seviyelerini ortaya çıkaran önemli bir araç olması ve ülkelerin karşılaştırılmasında önemli rol oynaması nedeniyle PISA puanları arasındaki bağımlılık yapısının incelenmesi önemlidir. Bu çalışmada 2006-2015 yılları arasındaki PISA fen, matematik ve okuma puanları için ikili bağımlılık yapıları en uygun kapula modeliyle belirlenmiş ve seçilen modellere göre bağımlılık yapıları yorumlanmıştır.
\end{abstract}

Anahtar Kelimeler: Kapula, PISA, OECD.

\section{PISA Modeling the Dependency Structure between Science, Mathematics and Reading Scores with Copula}

\begin{abstract}
PISA is a reliable study that measures the educational levels of students in science, mathematics and reading in a country. In this context, it is aimed to compare the educational levels of the countries and to determine the educational policies for the future by obtaining scores in different fields of the countries. PISA's translation and adaptation at national level, analysis and preparation of the national report are carried out by national centers designated for each participating country. Copulas are functions that represent the structure of dependence between variables and they form multivariate distributions. The main purpose of the copula function is to obtain the multivariate distribution, which is the most appropriate for the observed data, by revealing the dependency structure. It is important to examine the dependence structure between PISA scores, since PISA is an important tool to reveal the educational level of individuals and has an important role in comparing countries. In this study, the most appropriate paired dependence structures for PISA science, mathematics and reading scores between 2006 and 2015 were determined by the most suitable copula model and the dependency structures were interpreted according to the selected models.
\end{abstract}

Keywords: Copula, PISA, OECD

\section{Giriş}

Küreselleşen dünyada eğitimin amac1; bireylere bilgi öğretme, bu bilgileri kullanma ve bununla beraber bireyleri günlük hayatta karşılaştıkları yeni durumlara hazırlamaktır. $\mathrm{Bu}$ amaç doğrultusunda öğrencilerin sahip oldukları niteliklerin seviyesini ölçmek için OECD (Organization 
of Economic Cooperation and Development) tarafından, her üç yılda bir PISA (The Programme for International Student Assessment) araştırması yapılmaktadır. 2000 yılında başlayan PISA araştırmaları, araştırmaya katılan ülkelerdeki zorunlu eğitimi tamamlamış, 15 yaş üzerindeki bireyler üzerinde uygulanmaktadır. PISA, ülkelerarası öğrencilerin eğitim düzeylerinin karşılaştırılması ve eğitimde eksik yönlerin tespit edilmesinde önemli rol oynadığ için tüm dünyada devlet otoritelerinin ilgilendiği bir araştırmadır (Taş ve ark., 2016).

Araştırmada fen, matematik ve okuma alanlarında beceri puanları elde edilmektedir. PISA araştırmasında bu puanlar değerlendirilirken okuryazarlık kavramı esas alınmaktadır. Bu kavramda öğrencilerin değerlendirilecek olan alanda karşılaştıkları problemi tanımlayabilme, yorumlayabilme, anlayabilme, sahip oldukları bilgileri kullanarak problemin üstesinden gelebilme ve elde ettikleri sonuçlarla iletişim kurabilme becerileri ele alınmaktadır (Taş ve ark., 2016). Bu bağlamda fen, matematik ve okuma becerileri arasında ilişki olması beklenir. Değişkenler arasındaki ilişkinin anlaşılabilmesi için değişkenler arasındaki bağımlılık yapısının belirlenmesi gerekir. Değişkenler arasındaki bağımlılık yapısını modelleyen kapulalar, bir rastgele değişken vektörünün ortak dağılım fonksiyonu ile bu dağılımın marjinalleri arasında bağıntı kuran çok değişkenli, özel bir fonksiyondur (Alhan, 2008).

$\mathrm{Bu}$ çalışmada 2006-2015 yılları arasındaki PISA fen, matematik ve okuma beceri puanları baz alınmıştır. Fen, matematik ve okuma puanları birer değişken olarak ele alınmış ve bu değişkenler arasındaki bağımlılık yapısı, uygun kapula modelleri belirlenerek incelenmiş ve yıllara göre sonuçlar yorumlanmıştır.

\section{Materyal ve Yöntem}

Kapulalar değişkenler arasındaki bağımlılık yapısını belirlerken, bazı korelasyon ölçümleri ya da parametrik yöntemlerin sahip olduğu varsayımlara ihtiyaç duymadan, değişkenlerin ortak dağılım fonksiyonu ile marjinalleri arasında bağlantı kurar. $\mathrm{Bu}$ sayede, kapula ile değişkenler arasında bağımlılık yapısı belirlenirken diğer taraftan da değişkenlere uygun düşen çok değişkenli dağılım elde edilir. Kapulalar ile verilerin normal dağılmadığı ya da dağılımın bilinmediği durumlarda çok değişkenli bir modelle, bağımlılık yapısını yansıtan parametreler elde edilebilir.

Kapulalar, marjinal olasılık dağılımları için herhangi bir varsayım gerektirmeyen bir yaklaşım olup, bağımlı ve bağımsız değişken arasındaki lineer olmayan bağımlılığın modellenmesini sağlar. Ayrıca hiçbir korelasyon ölçüsü bu özelliğe sahip 
değildir. Dolayısıyla kapulalar, bağımlılık açısından ölçüden bağımsız olarak çalışmaya olanak sağlaması ve iki ya da daha fazla değişkene sahip dağılım ailelerini inşa etmede başlangıç noktası olması nedeniyle uygulamada önemli bir yere sahiptir (Nelsen, 2003).

Kapulaları matematiksel olarak aşağıdaki gibi ifade edebiliriz.

$$
C: I^{n} \rightarrow I
$$

$$
u \rightarrow C(u) \text { fonksiyonu, }
$$

$>\quad \forall u \in I^{n}$ için $u^{\prime}$ nun koordinatlarından en az biri 0 ise, $C(u)=0$

$>\quad \forall u \in I^{n}$ için $u_{k}$ hariç $u$ 'nun tüm koordinatları 1 ise, $C(u)=u_{k}$

$$
a \leq b \text { olan } \forall a, b \in I^{n} \text { için } V_{C}([a, b]) \geq 0
$$

şartlarını sağlıyorsa $C$ 'ye $n$-boyutlu kapula ya da kısaca $n$-kapula denir (Nelsen, 1999).

\subsection{Sklar Teoremi}

Kapulanın varlığını ortaya koyan bu teorem, ortak dağılım fonksiyonu ile kapulalar

arasındaki

bağıntıy1

tanımlamaktadır.

$H$, marjinalleri $F_{1}, F_{2}, \ldots, F_{n}$ olan n-boyutlu ortak dağ 1 lım fonksiyonu olsun. Bu takdirde $\forall x \in \bar{R}^{n}$ için,

$$
H\left(x_{1}, x_{2}, \ldots, x_{n}\right)=C\left(F_{1}\left(x_{1}\right), F_{2}\left(x_{2}\right), \ldots, F_{n}\left(x_{n}\right)\right)
$$

olacak şekilde bir $C$ n-kapulası vardır. Eğer $F_{1}, F_{2}, \ldots, F_{n}$ 'lerin hepsi sürekliyse o zaman $C$ tektir. Aksi takdirde $C$, $F_{1}, F_{2}, \ldots, F_{n}{ }^{\prime} l e r i n \quad$ değer kümelerinin kartezyen çarpımı üzerinde tek türlü tanımlanmıştır. Tersine $C$ bir n-kapula ve $F_{1}, F_{2}, \ldots, F_{n}$ dağılım fonksiyonları ise (2.2) eşitliğinde tanımlanan $H$ fonksiyonu, marjinalleri $F_{1}, F_{2}, \ldots, F_{n}$ olan n-boyutlu bir dağglım fonksiyonudur (Sklar, 1959).

$H$, marjinalleri $F_{1}, F_{2}, \ldots, F_{n}$ olan ve kapulası $C$ olan dağılım fonksiyonu olsun. $F_{1}^{(-1)}, F_{2}^{(-1)}, \ldots, F_{n}^{(-1)}$ sirasiyla $F_{1}, F_{2}, \ldots, F_{n}{ }^{\prime}$ lerin yarı tersleri olsun. $\mathrm{Bu}$ takdirde herhangi bir $u \in I^{n}$ için; 


$$
C\left(u_{1}, u_{2}, \ldots, u_{n}\right)=H\left(F_{1}^{(-1)}\left(u_{1}\right), F_{2}^{(-1)}\left(u_{2}\right), \ldots, F_{n}^{(-1)}\left(u_{n}\right)\right)
$$

eşitliği Sklar teoreminin bir sonucu olarak sağlanır.

\subsection{Kapula Aileleri}

\subsubsection{Clayton Kapula}

Clayton kapula bir Arşimedyen kapuladır. $\theta$ bağımlılık parametresi ile aşağıdaki gibi ifade edilir.

$$
C(u, v)=\left(u^{-\theta}+v^{-\theta}-1\right)^{-1 / \theta}, \quad 0 \leq \theta<\infty
$$

Clayton kapula tercih edilmelidir

$$
\theta \rightarrow 0 \text { iken } C(u, v)=\pi(u, v)=u v \quad \text { (Trivedi ve Zimmer, 2007). }
$$

olur ve bağımsızlık kapulasına ulaşılır. $\theta \rightarrow \infty$ iken mükemmel bağımlılığa işaret eder. Clayton kapula için sol kuyruk bağımlılı̆̆ı dikkate alınmalıdır. Yani, birlikte azalış göstermeye, birlikte artış göstermekten daha yatkın olan gözlemlerde

\subsubsection{Student's t Kapula}

Student's t kapula eliptik bir kapula olup, $r$ Pearson korelasyon katsayısı ve $t_{v}$ ise $v$ serbestlik dereceli student-t dağılımını belirtmek üzere bu iki bağımlılık parametresi ile aşağıdaki gibi ifade edilir.

$$
C\left(u_{1}, u_{2}\right)=\int_{-\infty}^{t_{v}^{-1}\left(u_{1}\right)} \int_{-\infty}^{t_{v}^{-1}\left(u_{2}\right)}\left(1+\frac{\left(s^{2}-2 r s t+t^{2}\right.}{v\left(1-r^{2}\right)}\right)^{-\frac{v+2}{2}} d s d t
$$

Burada $t_{v}^{-1}, v$ serbestlik derecesine sahip student $\mathrm{t}$ dağglımının tersini ifade eder. $v \rightarrow \infty$ için $C\left(u_{1}, u_{2}, r, v\right) \rightarrow \Phi_{G}\left(u_{1}, u_{2}, r\right)$ olur. Yani $v$ sonsuza yaklaştığında, Student's t kapula, Gaussian kapulaya yakınsar. Student's t kapula simetrik bağımlılık sergiler. Alt ve üst kuyruk bağımlılığı birbirine eşit olup $\lambda_{U}=\lambda_{L}=$ $2 t_{v+1}\left(\frac{-\sqrt{v+1} \sqrt{1-r}}{\sqrt{1+r}}\right) \quad$ ile ifade edilir (Wiboonpongse ve ark., 2015).

\subsubsection{Gaussian Kapula}

Gaussian kapula eliptik bir kapuladır. $\theta$ bağımlılık parametresi ile birlikte aşağıdaki gibi ifade edilir. 


$$
\begin{aligned}
C(u, v) & =\Phi_{G}\left(\Phi^{-1}(u), \Phi^{-1}(v) ; \theta\right) \\
& =\int_{-\infty}^{\Phi^{-1}(u)} \int_{-\infty}^{\Phi^{-1}(v)} \frac{1}{2 \pi \sqrt{1-\theta^{2}}} *\left(\frac{-\left(s^{2}-2 \theta s t+t^{2}\right)}{2\left(1-\theta^{2}\right)}\right) d s d t
\end{aligned}
$$

Burada $\Phi$ standart normal dağılım fonksiyonu, $\Phi_{G}(u, v)$ iki değişkenli standart normal dağılım fonksiyonudur. Gaussian kapulada $\theta$ bağımlılık parametresi, Pearson korelasyon ölçümü olup $[-1,1]$ aralığına kısıtlanmıştır. $\quad \theta \rightarrow 0$ için bağımsızlık kapulasına dönüşür. $\theta \rightarrow-1$ ve $\theta \rightarrow 1$ olduğunda sirasıyla Frechet alt sinır ve Frechet üst sınırına ulaşır. Eşit derecede pozitif ve negatif bağımlılığa izin verdiği için esnek bir kapula modelidir (Trivedi ve
Zimmer, 2007). Burada $u$ ve $v$ parametrik ya da non-parametrik keyfi bir dağılıma sahip olabilir. Ancak $u$ ve $v$ normal dağılan marjinallerse Gaussian kapula'da iki değişkenli dağılım fonksiyonu normal dağılıma sahiptir (Chen ve ark., 2017).

\subsubsection{Gumbel-Hougaard}

Kapula

Ailesi

Gumbel-Hougaard kapula bir Arşimedyen kapuladır. $\theta$ bağımlılık parametresi ile aşağıdaki gibi ifade edilir.

$$
C(u, v)=\exp \left\{-\left[(-\ln u)^{\theta}+(-\ln v)^{\theta}\right]^{1 / \theta}\right\}, 1 \leq \theta<\infty
$$

$\theta \rightarrow 1^{+}$için $C(u, v)=\pi(u, v)=u v$ bağımsızlık kapulasına dönüşür. Bu kapula hiçbir $\theta$ değeri için Frechet alt sınırına ulaşmaz. Gumbel kapula, güçlü sağ kuyruk bağımlılı̆̆ı sergiler. Bunun anlamı iki boyutlu rastgele değişkenler birlikte artış göstermeye, birlikte azalış göstermekten daha yatkındır. Küçük değerler için düşük korelasyon, yüksek değerler için güçlü korelasyona sahip gözlemler varsa, bu tür gözlemler için Gumbel kapula tercih edilmelidir. Gumbel kapula için sağ kuyruk bağımlılı̆̆ $\quad \lambda_{U}=2-2^{1 / \theta}$ ile hesaplanır (Trivedi ve Zimmer, 2007).

\subsubsection{Frank Kapula}

Frank kapula bir arşimedyen kapuladır. $\theta$ bağımlılık parametresi ile birlikte aşağıdaki gibi ifade edilir.

$$
C(u, v)=-\frac{1}{\theta} \ln \left[1+\frac{\left(e^{-\theta u}-1\right)\left(e^{-\theta v}-1\right)}{e^{-\theta}-1}\right], \theta \in R-\{0\}
$$




$$
\theta \rightarrow 0 \text { iken } C(u, v)=\pi(u, v)=u v
$$

bağımsızlık kapulasına ulaşılır. $\theta \rightarrow \infty$ iken Frechet üst sınırına $\theta \rightarrow-\infty$ iken Frechet alt sınırına eşit olur. Geniş bir parametre uzayına sahip olması ve marjinaller arasındaki negatif bağımlılı̆̆ın da modellenebilir olması nedeniyle, Frank kapula uygulamalarda daha çok tercih edilmektedir. Frank kapula ile modellenen gözlemler çok güçlü negatif ya da çok güçlü pozitif bağımlılığa sahiptir (Meester ve Mackay, 1994).

\subsection{Kapula Tahmini}

Rastgele değişkenler arasındaki bağımlılık yapısı kapula ile istatistiksel olarak, bir parametre ya da parametreler vektörü ile belirlenebilmektedir. Parametrelerin tahmin edilmesinde kullanılan maksimum olabilirlik yönteminde, tahmin edilen çok değişkenli dağ̊lımın, marjinal dağılımlarına ait parametreleri ile bağımlılık yapısını karakterize eden kapulaya ilişkin parametrelerin tamamı eşanlamlı olarak tahmin edilir (Joe, 1997).

Çok değişkenli bir dağılımın kanonik gösterimi aşağıdaki gibidir.

$$
\begin{gathered}
f\left(x_{1}, x_{2}, \ldots, x_{n}\right)=c\left(F_{1}\left(x_{1}\right), F_{2}\left(x_{2}\right), \ldots, F_{n}\left(x_{n}\right)\right) \prod_{j=1}^{n} f_{j}\left(x_{j}\right) \\
f_{j}\left(x_{j}\right)=\frac{\partial F_{j}\left(x_{j}\right)}{\partial x_{j}}, j=1, \ldots, n \text { ve } \\
c\left(F_{1}\left(x_{1}\right), F_{2}\left(x_{2}\right), \ldots, F_{n}\left(x_{n}\right)\right)=\frac{\partial^{n} C\left(F_{1}\left(x_{1}\right), F_{2}\left(x_{2}\right), \ldots, F_{n}\left(x_{n}\right)\right)}{\partial F_{1}\left(x_{1}\right) \partial F_{2}\left(x_{2}\right) \ldots \partial F_{n}\left(x_{n}\right)}
\end{gathered}
$$

olmak üzere, $c$ kapula yoğunluğu $C$ dağılımlar belirlenir ve daha sonra uygun kapulasının n. mertebeden kısmi türevini, $f_{j}$ 'ler ise marjinal olasıllk yoğunluk ark., 2004).

fonksiyonlarını ifade etmektedir. Örnek veri matrisi $S=\left\{x_{1 t}, x_{2 t}, \ldots, x_{n t}\right\}_{t=1}^{T}$ Kapulaların istatistiksel modellemesi iki olsun. Bu takdirde, log-olabilirlik adımda gerçekleşir. İlk olarak marjinal fonksiyonu aşağıdaki gibi ifade edilir.

$$
l(\theta)=\sum_{t=1}^{T} \ln \left\{c\left(F_{1}\left(x_{1 t}\right), F_{2}\left(x_{2 t}\right), \ldots, F_{n}\left(x_{n t}\right)\right)\right\}+\sum_{t=1}^{T} \sum_{j=1}^{n} \ln f_{j}\left(x_{j t}\right)
$$


Burada $\theta$, kapula ve marjinallere ilişkin parametreler vektörüdür. Marjinal dağılımlar ve kapula fonksiyonu biliniyorsa, yukarıdaki log-olabilirlik fonksiyonu yazılır ve maksimum olabilirlik tahmin edicisi $\hat{\theta}_{M L E}=\operatorname{maxl}(\theta)$ ile bulunur.

\subsection{Kapula Seçimi}

Farklı kapula fonksiyonları farklı bağımlılık modelleri sergiler. Bu nedenle, bağımlılık yapısı araştırılmak istenildiğinde, birkaç kapula modeli tahmin edilir ve verilere en uygun olanı seçilir (Trivedi ve Zimmer, 2005).

Maksimum olabilirlik fonksiyonu ile kullanılan AIC ya da BIC değerleri ile kapula seçimine karar verilir.

$$
B I C=-2 \ln (L)+k \ln (n)
$$

Burada $\ln (L)$ maksimize edilmiş logolabilirlik değeri, $k$ parametre sayısı, $n$ ise gözlemlerin sayısıdır. En küçük BIC değerine sahip kapula, bağımlılığı en iyi yansitan modeldir (Akaike, 1974).

$$
A I C=-2 \ln (L)+2 k
$$

Benzer şekilde, $\ln (L)$ maksimize edilmiş log-olabilirlik değeri, $k$ parametre sayısı olmak üzere en küçük AIC değerine sahip olan kapula, en iyi uyuma sahip kapuladır.

\section{Bulgular}

Bu çalışmada 31 ülkenin, 2006-2015 yıllarına ait PISA fen, matematik ve okuma puanları üzerinde araştırma yapılmıştır. Fen, matematik ve okuma puanları birer tesadüfi değişken olarak ele alınmış ve aralarındaki ikili bağımlılık yapıları için en uygun kapula modelleri tespit edilmiştir. Bağımlılık yapısını karakterize eden parametreler, parametrik yöntemlerden olan maksimum olabilirlik yöntemi ile elde edilmiştir. Elde edilen kapula modellerinden en uygun olanı AIC ve BIC değerlerine bakılarak minimum değere sahip olan model tercih edilmiştir. Tercih edilen kapula modeline göre ikili bağımlılık yapıları yıllara göre yorumlanmıştır.

2006-2015 yılları arasındaki FenMatematik, Fen-Okuma, Okuma-Matematik puanları arasındaki ikili bağımlılık yapıları incelendiğinde, kapula modellerine ait elde edilen AIC ve BIC değerleri Çizelge 1'de verilmiştir. En küçük AIC veya BIC değeri baz alınarak seçilen kapula modelleriyle puanlar arasındaki ikili bağımlılık yapıları belirlenmiştir. 
Çizelge 1. Fen-Matematik, Fen-Okuma, Okuma-Matematik puanları arasındaki ikili kapula modellerine ait elde edilen AIC ve BIC değerleri

\begin{tabular}{|c|c|c|c|c|}
\hline Ikili & Yil & Aile & AIC & BIC \\
\hline \multirow[t]{6}{*}{ FEN-MAT } & \multirow[t]{6}{*}{2006} & Gaussian & -57.71 & -56.28 \\
\hline & & Student's t & -55.7 & -52.83 \\
\hline & & Clayton & -75.48 & -74.04 \\
\hline & & Gumbel & -46.91 & -45.48 \\
\hline & & Frank & -52.59 & -51.16 \\
\hline & & Joe & -33.68 & -32.24 \\
\hline \multirow[t]{6}{*}{ FEN-OKUMA } & \multirow[t]{6}{*}{2006} & Gaussian & -33.01 & -31.58 \\
\hline & & Student's t & -32.41 & -29.55 \\
\hline & & Clayton & -39.27 & -37.83 \\
\hline & & Gumbel & -27.72 & -26.28 \\
\hline & & Frank & -30.17 & -28.74 \\
\hline & & Joe & -19.04 & -17.61 \\
\hline \multirow{6}{*}{ OKUMA-MAT } & \multirow[t]{6}{*}{2006} & Gaussian & -40.27 & -38.84 \\
\hline & & Student's t & -38.35 & -35.48 \\
\hline & & Clayton & -40.13 & -38.7 \\
\hline & & Gumbel & -35.67 & -34.23 \\
\hline & & Frank & -33 & -31.57 \\
\hline & & Joe & -27.73 & -26.3 \\
\hline \multirow[t]{6}{*}{ FEN-MAT } & \multirow[t]{6}{*}{2009} & Gaussian & -57.2 & -55.77 \\
\hline & & Student's t & -55.07 & -52.21 \\
\hline & & Clayton & -66.19 & -64.75 \\
\hline & & Gumbel & -47.53 & -46.1 \\
\hline & & Frank & -48.13 & -46.69 \\
\hline & & Joe & -35.85 & -34.42 \\
\hline \multirow[t]{6}{*}{ FEN-OKUMA } & \multirow[t]{6}{*}{2009} & Gaussian & -40.58 & -39.15 \\
\hline & & Student's t & -40.75 & -37.88 \\
\hline & & Clayton & -36.45 & -35.02 \\
\hline & & Gumbel & -38.92 & -37.48 \\
\hline & & Frank & -37.74 & -36.3 \\
\hline & & Joe & -32.25 & -30.81 \\
\hline \multirow[t]{6}{*}{ OKUMA-MAT } & \multirow[t]{6}{*}{2009} & Gaussian & -58.73 & -57.3 \\
\hline & & Student's t & -60.07 & -57.21 \\
\hline & & Clayton & -46.45 & -45.02 \\
\hline & & Gumbel & -61.89 & -60.46 \\
\hline & & Frank & -56.14 & -54.7 \\
\hline & & Joe & -56.51 & -55.08 \\
\hline \multirow[t]{6}{*}{ FEN-MAT } & \multirow[t]{6}{*}{2012} & Gaussian & -59.72 & -58.28 \\
\hline & & Student's t & -58.01 & -55.14 \\
\hline & & Clayton & -71.51 & -70.08 \\
\hline & & Gumbel & -49.2 & -47.77 \\
\hline & & Frank & -56.63 & -55.2 \\
\hline & & Joe & -35.75 & -34.32 \\
\hline \multirow[t]{6}{*}{ FEN-OKUMA } & \multirow[t]{6}{*}{2012} & Gaussian & -39.76 & -38.33 \\
\hline & & Student's t & -49.74 & -46.87 \\
\hline & & Clayton & -37.37 & -35.93 \\
\hline & & Gumbel & -45.47 & -44.04 \\
\hline & & Frank & -39.67 & -38.24 \\
\hline & & Joe & -39.98 & -38.54 \\
\hline \multirow[t]{6}{*}{ OKUMA-MAT } & 2012 & Gaussian & -41.78 & -40.35 \\
\hline & & Student's t & -40.8 & -37.94 \\
\hline & & Clayton & -37.98 & -36.55 \\
\hline & & Gumbel & -39 & -37.56 \\
\hline & & Frank & -39.38 & -37.95 \\
\hline & & Joe & -31.02 & -29.59 \\
\hline FEN-MAT & 2015 & Gaussian & -44.11 & -42.68 \\
\hline & & Student's t & -44.76 & -41.89 \\
\hline & & Clayton & -52.45 & -51.01 \\
\hline & & Gumbel & -38.81 & -37.38 \\
\hline & & Frank & -38.34 & -36.91 \\
\hline
\end{tabular}




\begin{tabular}{|c|c|c|c|c|}
\hline & & Joe & -29.29 & -27.86 \\
\hline \multirow[t]{6}{*}{ FEN-OKUMA } & \multirow[t]{6}{*}{2015} & Gaussian & -35.24 & -33.81 \\
\hline & & Student's t & -39.2 & -36.33 \\
\hline & & Clayton & -29.73 & -28.3 \\
\hline & & Gumbel & -36.74 & -35.31 \\
\hline & & Frank & -40.29 & -38.86 \\
\hline & & Joe & -30.76 & -29.33 \\
\hline \multirow[t]{6}{*}{ OKUMA-MAT } & \multirow[t]{6}{*}{2015} & Gaussian & -33.45 & -32.02 \\
\hline & & Student's t & -31.54 & -28.67 \\
\hline & & Clayton & -33.1 & -31.67 \\
\hline & & Gumbel & -26.72 & -25.28 \\
\hline & & Frank & -37.19 & -35.76 \\
\hline & & Joe & $-17,82$ & $-16,39$ \\
\hline
\end{tabular}

Fen-Matematik puanları arasındaki anlamı bireylere ait Fen-Matematik bağımlılık yapısına göre seçilen en uygun puanlarının, tüm yıllarda birlikte azalmaya kapula modelleri ve bu modellere ait eğilimli olduğu görülmektedir. Diğer bir parametreler Çizelge 2'de verilmiştir. Fenifadeyle bireylerin fen puanları azaldığında, Matematik puanları arasındaki bağımlılı̆̆ matematik puanlarının da azaldığını benzer en iyi modelleyen Clayton kapula ailesi şekilde matematik puanları azaldığında, fen olmuştur. Bu aileye ait parametreler; 2006 puanlarının da azaldığını söylemek y1lı için 8.207788, 2009 y1lı için 6.610368, mümkündür. Y1llara göre Fen-Matematik 2012 y1lı için 7.743329 ve 2015 y1lı için puanları arasındaki bağımlılık yapısı Şekil 5.098275 olarak elde edilmiştir. Bunun 1’deki gibi gösterilebilir.

Çizelge 2. Fen-Matematik puanları arasında seçilen en uygun kapula modelleri ve bu modellere ait parametreler

\begin{tabular}{|l|}
\hline Ikililer \\
\hline FEN-MAT \\
\hline FEN-MAT \\
\hline FEN-MAT \\
\hline FEN-MAT \\
\hline
\end{tabular}

\begin{tabular}{|l|l|}
\hline Yillar & Aileler \\
\hline 2006 & Clayton \\
\hline 2009 & Clayton \\
2012 & Clayton \\
2015 & Clayton \\
\hline
\end{tabular}
par1 8.207788 

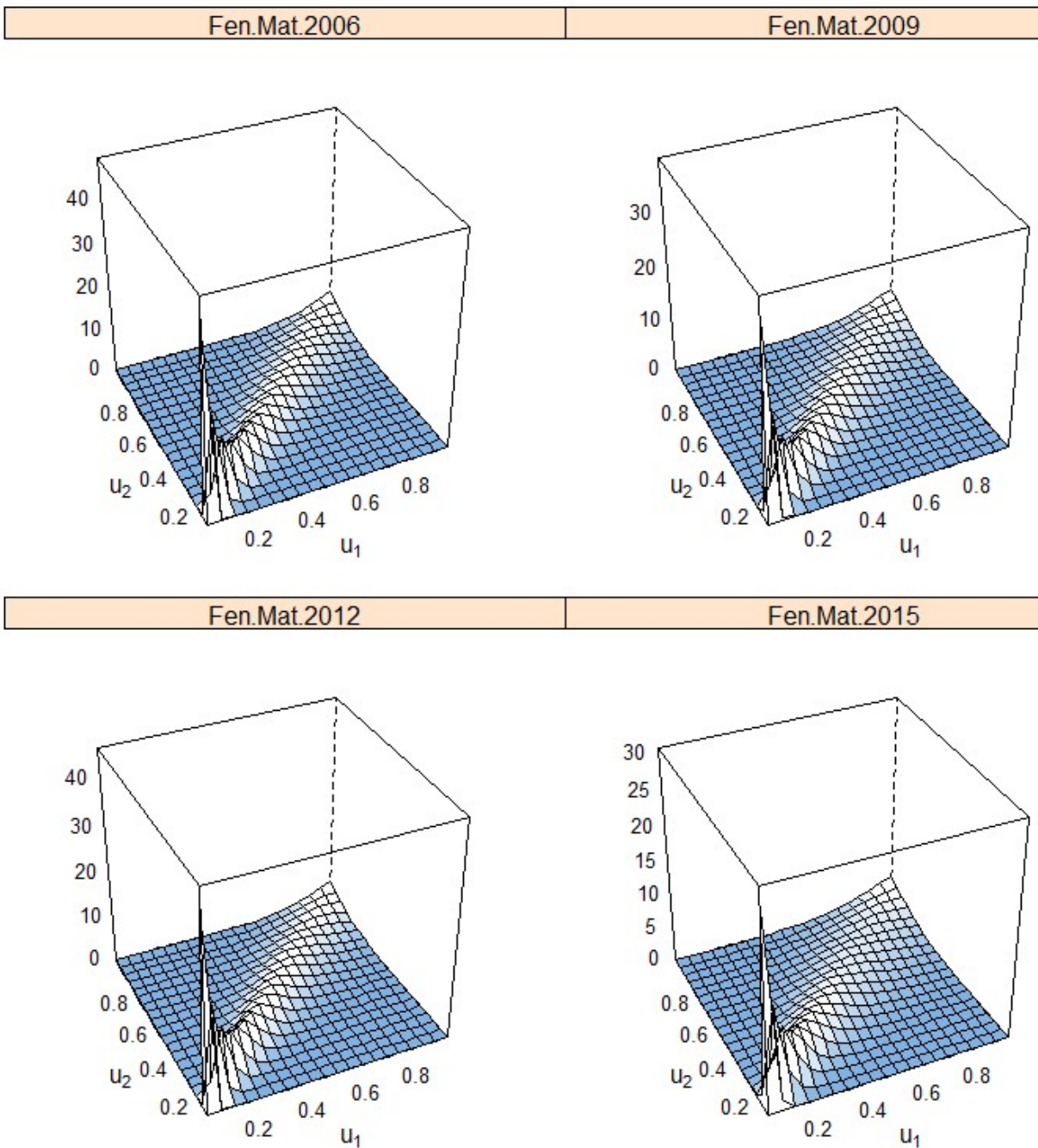

\begin{tabular}{l|r} 
Fen.Mat.2012 & Fen.Mat.2015
\end{tabular}

Şekil 1. Yıllara göre Fen-Matematik puanları arasındaki bağımlılık yapıları

Fen-Okuma puanları arasındaki bağımlılık yapısına göre seçilen en uygun kapula modelleri ve bu modellere ait parametreler Çizelge 3'de verilmiştir. 2006 yılında Fen-Okuma puanları arasındaki bağımlılı̆̆ı en iyi modelleyen Clayton kapula ailesi olmuştur. Fen-Okuma puanlarının birlikte azalmaya eğilimli olduğu görülmektedir. Diğer bir ifadeyle bireylerin fen puanları azaldığında, okuma puanlarının da azaldığını benzer şekilde okuma puanları azaldığında, fen puanlarının

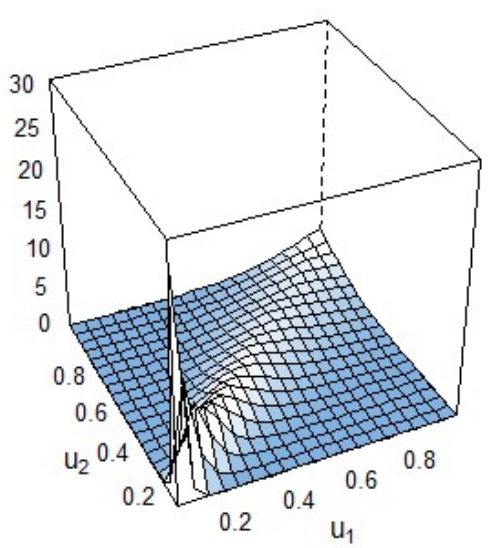

da azaldığını söylemek mümkündür. 2009 yılı için, Fen-Okuma puanları arasında bağımlılı̆̆1 en iyi modelleyen Gaussian kapula parametresi 0.885637 olarak bulunmuştur. Fen-Okuma puanları arasında güçlü pozitif bağımlılık söz konusudur. Bunun anlamı, Fen ve okuma puanlarının biri artarken diğerinin artma, biri azalırken diğerinin azalma eğiliminde olduğunu söylemek mümkündür. 2012 yılı için, FenOkuma puanları arasında bağımlılığı en iyi modelleyen Student's t kapula parametresi 
0.918422 olarak bulunmuștur. Fen-Okuma puanları arasında güçlü bir simetrik bağımlılık söz konusudur. 2015 y1lı için, Fen-Okuma puanları arasında bağımlılı̆ğ en iyi modelleyen Frank kapula parametresi 11.618 olarak bulunmuştur. Fen-Okuma puanları arasında diğer yıllara kıyasla daha güçlü pozitif bağımlılık söz konusudur. Yıllara göre Fen-Okuma puanları arasındaki bağımlılık yapısı Şekil 2'deki gibi gösterilebilir. parametreler

Çizelge 3. Fen-Okuma puanları arasında seçilen en uygun kapula modelleri ve bu modellere ait

\begin{tabular}{|l|l|l|l|l|l|}
\hline Ikililer & Yllar & Aileler & par1 & par2 & tau \\
\hline FEN-OKUMA & 2006 & Clayton & 3.489343 & 0 & 0.635658 \\
\hline FEN-OKUMA & 2009 & Gaussian & 0.885637 & 0 & 0.692555 \\
\hline FEN-OKUMA & 2012 & $\mathrm{t}$ & 0.918422 & 2.0001 & 0.741073 \\
\hline FEN-OKUMA & 2015 & Frank & 11.618 & 0 & 0.70445 \\
\hline
\end{tabular}

\begin{tabular}{|c|c|}
\hline Fen.Okuma.2006 & Fen.Okuma.2009 \\
\hline
\end{tabular}
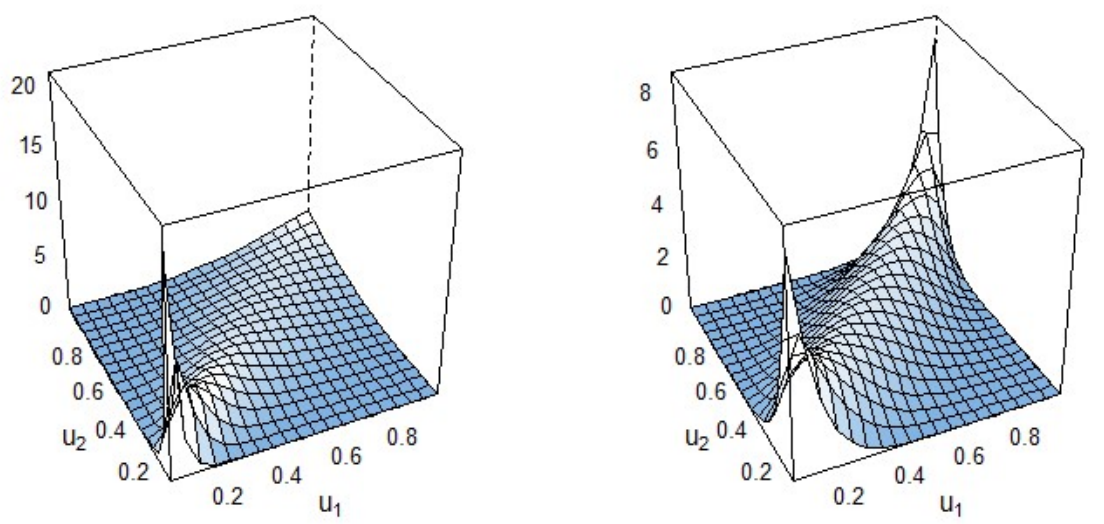

Fen.Okuma.2012
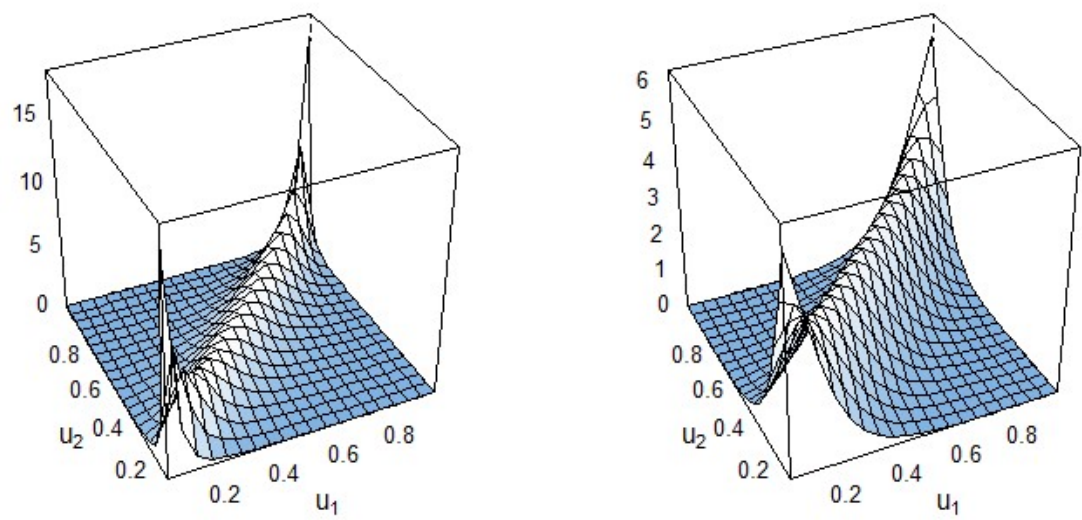

Şekil 2. Yıllara göre Fen-Okuma puanları arasındaki bağımlılık yapıları 
Okuma-Matematik puanları bağımlılık yapısına göre seçilen en uygun kapula modelleri ve bu modellere ait parametreler Çizelge 4'de verilmiştir. 2006 yılı için, Okuma-Matematik puanları arasında bağımlılığı en iyi modelleyen Gaussian kapula parametresi 0.884366 olarak bulunmuştur. Okuma-Matematik puanları arasında güçlü pozitif bağımlılık söz konusudur. Bunun anlami, Okuma ve Matematik puanlarının biri artarken diğerinin artma, biri azalırken diğerinin azalma eğiliminde olduğunu söylemek mümkündür. 2009 yılı için, OkumaMatematik puanları arasında bağımlılığı en iyi modelleyen Gumbel kapula parametresi 4.586192 olarak bulunmuştur. OkumaMatematik puanlarının birlikte artmaya eğilimli olduğu görülmektedir. Diğer bir ifadeyle bireylerin okuma puanları arttığında, matematik puanlarının da arttığı benzer şekilde matematik puanları arttığında, okuma puanlarının da arttığını söylemek mümkündür. 2012 y1lı için, Okuma-Matematik puanları arasında bağımlılı̆g en iyi modelleyen Gaussian kapula parametresi 0.890511 olarak bulunmuştur. Okuma-Matematik puanları arasında güçlü pozitif bağımlılık söz konusudur. 2015 y1lı için, OkumaMatematik puanları arasında bağımlılığı en iyi modelleyen Frank kapula parametresi 10.40644 olarak bulunmuştur. Yıllara göre Okuma-Matematik puanları arasındaki bağımlılık yapısı Şekil 3'deki gibi gösterilebilir.

Çizelge 4. Okuma-Matematik puanları arasında seçilen en uygun kapula modelleri ve bu modellere ait parametreler

\begin{tabular}{|l|l|l|l|l|l|}
\hline $\begin{array}{l}\text { Io:kililer } \\
\text { OKUMA-MAT }\end{array}$ & Yllar & Aileler & par1 & par2 \\
\hline OKUMA-MAT & 2006 & Gaussian & 0,884366 & 0 & 0,690818 \\
\hline OKUMA-MAT & 2009 & Gumbel & 4,586192 & 0 & 0,781954 \\
\hline OKUMA-MAT & 2012 & Gaussian & 0,890511 & 0 & 0,699306 \\
\hline
\end{tabular}



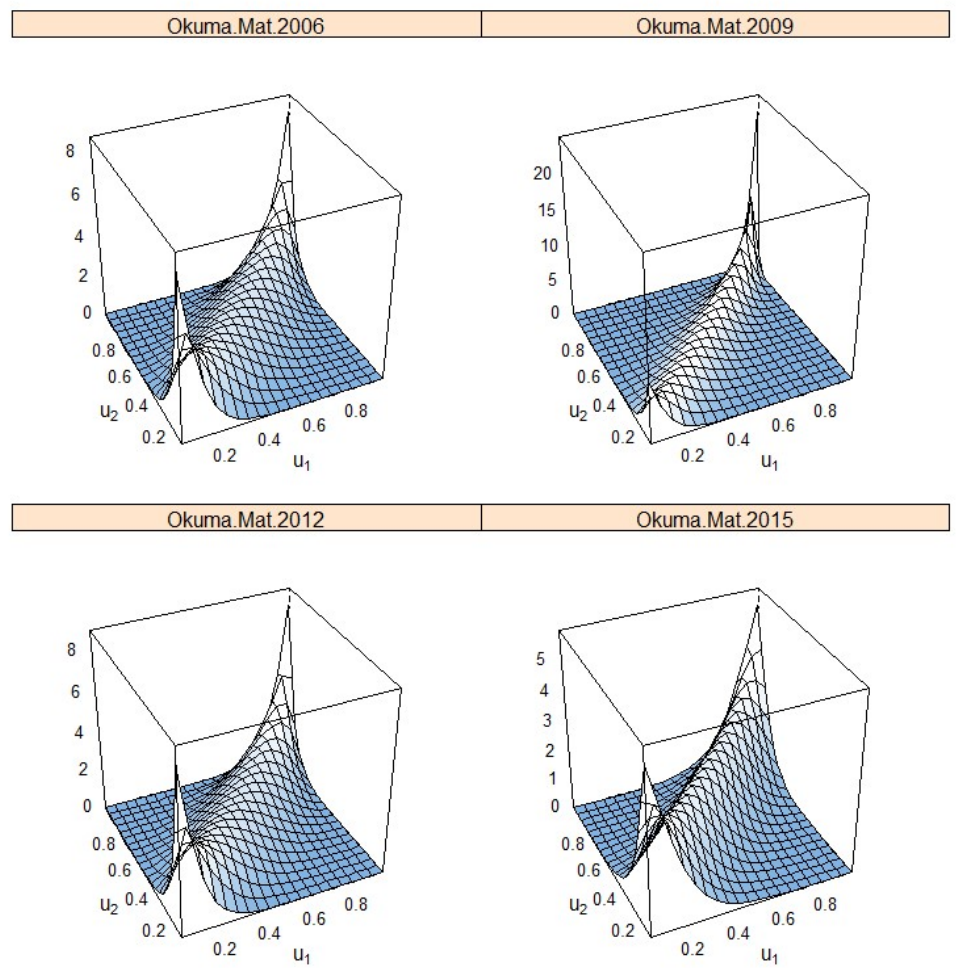

Şekil 3. Yıllara göre Okuma-Matematik puanları arasındaki bağımlılık yapıları

\section{Tartışma}

PISA 2006-2015 yılları arasındaki Fen, Matematik ve Okuma puanlarının ikili bağımlılık yapıları kapula modelleri ile belirlenmiş ve en uygun kapula modelinin seçimine AIC ve BIC değerlerine bakılarak karar verilmiştir.

Fen-Matematik puanlarının tüm yıllarda sol kuyruk bağımlılığına sahip olduğu ve 2015 y1lında Fen-Matematik puanları arasında bağımlılı̆̆ın en az, 2006 y1lında ise en fazla olduğu görülmektedir. 2006 y1lı Fen-Okuma puanlarının sol kuyruk bağımlılığg sergilediği ve 2015 yılı FenOkuma puanlarının diğer yıllara kıyasla daha güçlü bağımlılık yapısına sahip olduğu görülmektedir. 2009 y1lı Okuma-Matematik puanlarının sağ kuyruk bağımlılığ sergilediği ve 2015 yılı Okuma-Matematik puanları arasında diğer yıllara kıyasla daha güçlü pozitif bağımlılık yapısına sahip olduğu elde edilmiştir.

Sonuçlar değerlendirildiğinde, FenOkuma ve Okuma-Matematik arasındaki bağımlılığın son yıllarda daha da arttığ1 görülmektedir. Ülkeler bireylerin okuma becerilerini geliştirdiğinde ve bununla ilgili eğitim politikalarına yön verdiğinde, Matematik ve Fen alanında başarının daha da artacağını söyleyebiliriz. 


\section{Kaynaklar}

Akaike H (1974). A new look at the statistical model identification. In Selected Papers of Hirotugu Akaike, Springer, New York, 215-222.

Alhan A (2008). Bağımsızlık kapulasını içeren kapula aileleri, kapula tahmin yöntemleri ve İstanbul Menkul Kıymetler Borsasında sektörler arası bağımlılık yapısı. Doktora Tezi, Gazi Üniversitesi Fen Bilimleri Enstitüsü İstatistik Anabilim Dal, 162, Ankara.

Cherubini U, Luciano E, Vecchiato W (2004). Copula methods in finance. John Wiley and Sons, 289, New York.

Chen M, Yu G, Chen P, Wang Y (2017). A copula-based approach for estimating the travel time reliability of urban arterial. Transportation Research Part C: Emerging Technologies 82: 1-23.

Joe H (1997). Multivariate models and multivariate dependence concepts. CRC Press 395, London.

Meester SG, Mackay J (1994). A parametric model for cluster correlated categorical data. Biometrics 954-963.

Nelsen RB (2003). Properties and applications of Copulas: A Brief Survey. In Proceedings of the First Brazilian Conference on Statistical Modeling in Insurance and Finance, September, University Press USP: Sao Paulo, Brazil, pp. 10-28.

Nelsen RB (1999). An introduction to Copulas. Springer, New York, 1-4.

Sklar A (1959). Fonctions de Répartition à n Dimensions et Leurs Marges. Publ Inst Statist Univ 8: 229-231.

Trivedi PK, Zimmer DM (2005). Copula modeling: An introduction for practitioners. Publishers Inc., 28, Hanover, USA.

Trivedi PK, Zimmer DM (2007). Copula modeling: An introduction for practitioners. Foundations and Trends in Econometrics 1(1): 1-111.

Taş UE, Arıcı Ö, Ozarkan HB, Özgürlük B (2016). PISA 2015 ulusal raporu. Ankara: Milli Ĕ̈itim Bakanlığl.

Wiboonpongse A, Liu J, Sriboonchitta S, Denoeux T (2015). Modeling dependence between error components of the stochastic frontier model using Copula: Application to intercrop coffee production in Northern Thailand. International Journal of Approximate Reasoning 65: 34-44.

URL-1: http://www.oecd.org/pisa/data/ (Erişim Tarihi: 29.06.2017) 